\title{
Introduction to Processing of CT Clinical Metadata of Disabled Part of Patient Knee Joint
}

Josef Sedlak, Josef Chladil, Martin Slany, Karel Kouril

Department of Machining Technology, Institute of Manufacturing Technology, Faculty of Mechanical Engineering, Brno University of Technology, Technická 2896/2, Brno 616 69, Czech Republic. E-mail: sedlak@fme.vutbr.cz, chladil@fme.vutbr.cz, slany.m@fme.vutbr.cz, kouril.k@fme.vutbr.cz

This article focuses on an analysis and a transfer process of $\mathrm{CT}$ clinical metadata of a real patient gained within a cooperation, research and development of an individual knee joint implant at Clinique of Display Methods in Saint Anna's Teaching hospital in Brno. The first part of the article is aimed at the application of a software 3DDOCTOR that enables gaining of demanded output data (e.g. a model of patient bone part, i.e. knee joints) from scanned input CT metadata in DICOM format (Digital Imaging and Communications in Medicine). The output data are gained in format *.stl (Stereo Lithography) to further possible usage (e.g. a design and a production of individual total joint prosthesis). The second part of the article concentrates on an application of software RP MiniMagics that enables editing, modification and the whole optimization of polygon net by which models of distal part of femur and proximal part of tibia are described.

Keywords: Knee Joint, CT Metadata, 3D-DOCTOR, Technology, RP MiniMagics

\section{Acknowledgement}

The research was supported and co-financed from the project called "Excellent young scientists at VUT University in Brno" - register number CZ.1.07/2.3.00/30.0039.

\section{References}

[1] NEDOMA, J., et al. Biomedicíncká informatika II: Biomechanika lidského skeletu a umělých náhrad jeho částí. [online]. 2006 [vid. 15. dubna 2013]. Available at: http://ucebnice.euromise.cz/index.php?conn=0\&section=biomech. ISBN 80-246-1227-5.

[2] SEDLÁK, J., CHARVÁT, O., MADAJ, M. Technology of processing CT data of the Knee Joint. Manufacturing TECHNOLOGY, 2011, roč. X, č. 1, s. 64-70. ISSN 1213-2489.

[3] Počitačová tomografie a Hounsfieldovy jednotky. [online]. 2013 [vid. 20. zááí 2013]. Available at: http://www.wikiskripta.eu/index.php/Po\%C4\%8D\%C3\%ADta\%C4\%8Dov\%C3\%A1_tomografie_a_Hounsfieldovy_jednotky.

[4] Počitačová tomografie. [online]. 2013 [vid. 20. září 2013]. Available at: http://cs.wikipedia.org/wiki/Po\%C4\%8D\%C3\%ADta\%C4\%8Dov\%C3\%A1_tomografie.

[5] 3D-DOCTOR 4.0.200800808. [online]. ${ }^{\circ}$ 1998-2008. Able Software Corp. All rights reserved. [vid. 8. ledna 2012]. Available at: http://www.ablesw.com/3d-doctor.

[6] TomoCon PACS. [online]. ${ }^{\circledR} 2012$ TatraMed spol. s r.o. Všetky práva vyhradené. [vid. 12. dubna 2012]. Available at: http://www.tatramed.com/pacsItem?element=3\&parentId=7\&type=13.

[7] STL Model Creator. [online]. ${ }^{\circ}$ Biomechanika 2. Brno: Vysoké učení technické v Brně, Fakulta strojního inženýrství, Ústav mechaniky těles, mechatroniky a biomechaniky, 2010. [vid. 10. června 2012]. Available at: http://biomechanika.fme.vutbr.cz/index.php?option=com_content\&view=article\&id=59\%3Astl-model-creator\&catid $=36 \% 3$ Asoftware\&Itemid $=62 \&$ lang $=$ cs.

[8] Text tématu - E-learning. [online]. (C) 2007 [vid. 14. dubna 2012]. Př́íprava vstupních geometrických dat pro MKP softwary. Available at: http://blade1.ft.tul.cz/ tyr/cgibin/elearning/elear-

ning.fcgi?page $=$ publ\&action $=$ showThemeContentText\&item $=421 \&$ theme_id $=36$.

[9] Materialise NV. [online]. ${ }^{\circ}$ 2012. Software for Additive Manufacturing. [vid. 14. zář́ 2012]. MiniMagics - Software Rapid Prototyping. Available at: http://www.materialise.com/MiniMagics.

[10] 3Dim Laboratory s.r.o. [online]. @ 2008 [vid. 20. dubna 2012]. Tvorba 3D modelů tkání na základě CT/MR dat. Available at: http://www.3dim-laboratory.cz/en/products/cranioform.

[11] SYNBONE anotomical models for education. [online]. 2008 [vid. 20. dubna 2012]. Mechanical properties. Available at: http://www.synbone.ch/wEnglish/products/mechanical_properties.php?navanchor=1010003. 
[12] Zdravotnictví / Protocom - 3D tisk, 3D tiskárny, ZCorporation, 3D Copycentrum, 3D Scan, Rapid Prototyping, $3 D$ Systems. [online]. 2008 [vid. 20. dubna 2012]. Předoperační plánování. Available at: http://www.protocom.cz/reseni/zdravotnictvi/predopracni-planovani/.

[13] PAPACHRISTOU, G. (2004). Photoelastic study of the internal and contact stresses on the knee joint before and after osteotomy Journal. Archives of Orthopaedic and Trauma Surgery. Volume 124, Number 5/June, p. 533.

[14] CHARVÁT, O., SEDLÁK, J., MADAJ, M. (2009). New Technique during Production of Knee Point Replacements. Strojírenská technologie, roč. XIV, č. 2, s. 34-39. ISSN: 1211-4162.

\section{Paper number: M2014113}

Copyright (C) 2014. Published by Manufacturing Technology. All rights reserved. 